\title{
MAXIMAL QUOTIENT RINGS OF PRIME GROUP ALGEBRAS. II UNIFORM RIGHT IDEALS
}

\author{
JOHN HANNAH \\ (Received 16 August 1976; revised 4 November 1976) \\ Communicated by M. F. Newman
}

\begin{abstract}
Suppose $K G$ is a prime nonsingular group algebra with uniform right ideals. We show that $G$ has no nontrivial locally finite normal subgroups. If $G$ is soluble or residually finite, or if $K$ has zero characteristic and $G$ is linear, then the maximal right quotient ring of $K G$ is simple Artinian.
\end{abstract}

\section{Introduction}

It was shown in Hannah and O'Meara (to appear) that if a prime nonsingular group algebra $K G$ has no uniform right ideals, where $G$ is a group whose conjugacy classes are countable, then the maximal right quotient ring of $K G$ is simple and directly infinite. Suppose now that $K G$ is prime nonsingular and does have uniform right ideals, and let $Q$ be its maximal right quotient ring. In this case a well-known result of Johnson (1961) says that $Q$ is the ring of all linear transformations of a vector space over some division ring. Hence either $Q$ is simple Artinian (which happens exactly when $K G$ has finite uniform dimension), or $Q$ is directly infinite and not simple (the socle being a nonzero proper ideal). We shall show however that if $G$ is, for example, soluble or residually finite, or if $K$ has zero characteristic and $G$ is linear, then $Q$ must be simple Artinian. In particular it follows that a prime nonsingular group algebra (with or without uniform right ideals) always has a simple maximal right quotient ring when the group is of one of these types and has only countable conjugacy classes.

In section 1 we show that because $Q$ is a full-linear ring $G$ cannot have nontrivial locally finite normal subgroups. This condition has also arisen in the work of Handelman and Lawrence (1975), and they conjectured that it is equivalent to having the group algebra strongly prime. If this were so then it 
would follow that $Q$ is always simple Artinian. We show that when $G$ is soluble, Handelman and Lawrence's conjecture is true. In section 2 we suppose we have a normal subgroup $H$ of $G$ whose group algebra has a simple Artinian maximal right quotient ring. Then if $G / H$ is residually finite or locally finite it is shown that $Q$ too is simple Artinian. Finally in section 3 we use these results to give the above-mentioned result for linear groups.

NOTE. For rings we retain the terminology of Hannah and O'Meara (to appear) while for groups we follow the usage of Robinson (1972). In what follows $K$ denotes a field, $G$ a group, $Q(K G)$ the maximal right quotient ring of the group algebra $K G$ and $Z(K G)$ the (right) singular ideal of $K G$. For the properties of the singular ideal and quotient rings see Faith (1967) and Johnson (1961). If $X$ is a subset of a ring $R$ we denote the left (right) annihilator of $X$ in $R$ by $l(X)$ (respectively, $r(X)$ ).

\section{Normal subgroups}

In this section we look at a prime nonsingular group algebra $K G$ with uniform right ideals and see what effect these hypotheses have on the normal subgroups of $G$. Our main result (theorem 5) is that such subgroups cannot be locally finite. Corollary 6 shows that the group algebra of a subnormal subgroup of $G$ is also prime nonsingular with uniform right ideals. Firstly we need the following technical results.

LEMMA 1. Let $H$ be a subgroup of $G$ such that, for any finite subset $X$ of $G$, $H$ is ascendant in $\langle H, X\rangle$. Then

(a) any large (that is, essential) right ideal of $K H$ generates a large right ideal of $K G$ and so $Z(K H) \subseteq Z(K G)$;

(b) any dense right ideal of $K H$ generates a dense right ideal of $K G$ and so there is a natural embedding of $Q(K H)$ into $Q(K G)$ from which we get the identification

$$
Q(K H)=\{q \in Q(K G):\{\alpha \in K H: q \alpha \in K H\} \text { is a dense right ideal of } K H\} .
$$

ProOf. When $H$ is subnormal in $G$ the result about large right ideals is proved by Burgess (1969), while the result about dense right ideals is proved by Formanek (1974). This can be extended to ascendant subgroups by transfinite induction and then the result follows easily.

Throughout this paper we shall identify $Q(K H)$ with this subring of $Q(K G)$ whenever $H$ satisfies the hypothesis of the lemma.

Lemma 2. Suppose $H \triangleq G$ and let $\left\{x_{i}: i \in I\right\}$ be a transversal for $H$ in $G$. Then 
(a) for any $g \in G$ the function $\alpha \rightarrow g \alpha g^{-1}$ is an automorphism of $Q(K H)$;

(b) the subring of $Q(K G)$ generated by $Q(K H)$ and $K G$ is $\sum_{i \in I} Q(K H) x_{i}$ which is a free $Q(K H)$-module with normalizing basis $\left\{x_{i}: i \in I\right\}$.

Proof. Using lemma 1 and the fact that $H \leqslant G$ gives (a) and then (b) follows easily.

Because of lemma 2(b) we shall often denote by $Q(K H) \cdot G$ the subring of $Q(K G)$ generated by $Q(K H)$ and $K G$. Our first main result illustrates the advantages of being able to move up to the maximal right quotient rings of $K H$ and $K G$ when $H$ is normal in $G$.

THEOREM 3. Suppose $H \leqslant G$. If $K G$ is nonsingular and has a uniform right ideal then so does $\mathrm{KH}$.

Proof. For clarity's sake we introduce the temporary notation $R=$ $Q(K H), S=Q(K H) \cdot G$ and $Q=Q(K G)$. Since $K G$ is nonsingular $Q$ is regular. As $Q$ is a right quotient ring of $K G$, a uniform right ideal of $K G$ generates a uniform right ideal of $Q$. But uniform right ideals of $Q$ are just minimal right ideals because $Q$ is regular. Hence we can find $0 \neq \alpha \in S$ such that $\alpha Q$ is a minimal right ideal of $Q$. Write $\alpha=\alpha_{1} x_{1}+\cdots+\alpha_{n} x_{n}$ where each $0 \neq \alpha_{i} \in R$ and $x_{1}, \cdots, x_{n}$ are elements of some transversal for $H$ in $G$. We may suppose that $x_{1}=1$ and that $n$ is minimal for all these properties. Hence we have $l\left(\alpha_{1}\right)=l\left(\alpha_{2}\right)=\cdots=l\left(\alpha_{n}\right)$ in $R$ else we could reduce $n$. It is enough to show that $\alpha_{1} R$ is minimal so suppose it is not minimal.

By lemma 1(a), $K H$ is nonsingular and so $R$ is regular. Hence for some $\beta \in R$ we have $\alpha_{1} \beta \neq 0$ and $l\left(\alpha_{1}\right) \subset l\left(\alpha_{1} \beta\right)$ in $R$. Choose $\gamma \in R$ such that $\gamma \alpha_{1} \beta=0$ and $\gamma \alpha_{1} \neq 0$. Then

$$
\alpha \beta=\alpha_{1} \beta+\alpha_{2} \beta_{2} x_{2}+\cdots+\alpha_{n} \beta_{n} x_{n}
$$

where each $\beta_{i}=x_{i} \beta x_{i}^{-1} \in R$ by lemma 2 (a). Since $\alpha_{1} \beta \neq 0$ we have $\alpha \beta \neq 0$ by lemma 2(b) and so $\alpha \beta Q=\alpha Q$ is minimal. By the minimality of $n$ we get $l\left(\alpha_{1} \beta\right)=l\left(\alpha_{2} \beta_{2}\right)=\cdots=l\left(\alpha_{n} \beta_{n}\right)$ in $R$. As $\gamma \alpha_{1} \beta=0$ we thus have $\gamma \alpha \beta=0$ and so $\gamma \alpha=0$ because $\alpha \beta Q=\alpha Q$. But $\gamma \in R$ and $\gamma \alpha=\gamma \alpha_{1}+\gamma \alpha_{2} x_{2}+\cdots+\gamma \alpha_{n} x_{n}$ and so, by lemma $2(\mathrm{~b}), \gamma \alpha_{1}=0$ which is a contradiction. This completes the proof.

To apply this result to prime group algebras we need the following lemma.

Lemma 4. Suppose $G$ is locally finite with no finite nontrivial characteristic subgroups. If $K G$ is nonsingular then it has no uniform right ideals. 
Proof. By proposition 9 of Snider (to appear) $K G$ is semisimple. As in lemma 2.1 of Fisher and Snider (1974) every nonzero right ideal contains a nonzero idempotent. Hence we just have to show that $\operatorname{soc} K G=0$. But if soc $K G \neq 0$ then by theorem 5 of Hartley and Richardson (submitted) $G$ is a Cernikov group and so has a finite nontrivial characteristic subgroup, which is a contradiction.

TheOREM 5. If $K G$ is prime nonsingular with uniform right ideals then $G$ has no nontrivial locally finite normal subgroups.

ProOf. If $H$ is a nontrivial locally finite normal subgroup of $G$ it has no finite nontrivial characteristic subgroups because $G$ is prime (that is, has no finite normal nontrivial subgroups). By lemma $4, K H$ has no uniform right ideals. This contradicts theorem 3 .

Corollary 6. Suppose $H$ is a subgroup of $G$ such that, for any finite subset $X$ of $G, H$ is ascendant in $\langle H, X\rangle$ (for instance, $H$ could be subnormal in $G$ ). If $K G$ is prime nonsingular with uniform right ideals then so is $K H$.

Proof. Suppose first that $H \unlhd G$. Then $K H$ is nonsingular by lemma (a), and has uniform right ideals by theorem 3. Since $\Delta^{+} H \leqslant G, H$ is prime by theorem 5. By induction the corollary holds if $H$ is subnormal in $G$. Using transfinite induction we extend it further to the case where $H$ is ascendant in $G$ since the normal closure of an ascendant locally finite subgroup is again locally finite. The rest of the result follows easily.

We recall that a ring $R$ is said to be (right) strongly prime if for any nonzero $a \in R$ there is a finite subset $S(a)$ of $R$ such that $r(\{a s: s \in S(a)\})=$ 0 , or (equivalently) if every nonzero (two-sided) ideal of $R$ contains a finite subset whose right annihilator is zero. Any strongly prime ring with uniform right ideals has a simple Artinian maximal right quotient ring (see Handelman and Lawrence (1975) Corollary 1, page 218).

Handelman and Lawrence show that if the group algebra $K G$ is strongly prime then $G$ has no nontrivial locally finite normal subgroups, and they conjecture that the converse is also true. This is clearly so if $G$ is locally nilpotent since then the torsion elements of $G$ form a locally finite normal subgroup of $G$ and since the group algebra of a torsion-free nilpotent group is a domain. Another case is given by the following result.

Proposition 7. Suppose $G$ is soluble. The following statements are equivalent:

(a) $K G$ is strongly prime;

(b) $G$ has no nontrivial locally finite normal subgroups; 
(c) the Zalesskit subgroup of $G$ (see page 81 of Passman (1974)) is torsion-free.

ProOF. a) implies b): is proved by Handelman and Lawrence (1975).

b) implies c): follows because the Zalesskil subgroup of $G$ is an $F C$-group normal in $G$.

c) implies a): if $I \neq 0$ is an ideal of $K G$ and $H$ is the Zalesskir subgroup of $G$ then $I$ contains a nonzero element of $K H$ (see Zalesskiǐ (1973)). By hypothesis $K H$ is a domain and so $I$ contains a non-zerodivisor. Hence $K G$ is strongly prime.

An immediate corollary to this and theorem 5 is:

COROL.LARY 8. Suppose $G$ is soluble or locally nilpotent. If $K G$ is prime nonsingular with uniform right ideals then $Q(K G)$ is simple Artinian.

\section{Subgroups of finite index}

If $K G$ is prime nonsingular with uniform right ideals and $H \leqslant G$ then. by corollary 6, $Q(K H)$ is at least a full-linear ring. Suppose $Q(K H)$ is simple Artinian. By studying subgroups of finite index we show (corollary 12) that $Q(K G)$ too is simple when $G / H$ is residually finite. If on the other hand $G / H$ is locally finite we construct a dimension function on $Q(K H) \cdot G$ and again show that $Q(K G)$ is simple (theorem 15$)$. We begin with another technical result.

LFmma 9. Suppose $H \exists G$. (a) If $[G: H]<x$ then $Q(K H) \cdot G=Q(K G)$ and if in addition $K H$ is nonsingular then $Q(K G)$ is right self-injective.

(b) If $[G: H]<x$ and $K G$ is nonsingular then $Q(K H) \cdot G$ is regular.

(c) If $A$ is a right $Q(K H)$-submodule of $Q(K G)$ and $g \in G$ then $A g=\{a g: a \in A\}$ is a right $Q(K H)$-submodule with its lattice of submodules isomorphic to that of $A$.

Proof. (a) That $Q(K H) \cdot G=Q(K G)$ follows as in (3.2) of Burgess (1969). If $K H$ is nonsingular then $Q(K H)$ is right self-injective and hence so is $Q(K H) \cdot G=Q(K G)$ (see (2.8) of Burgess (1969)).

(b) now follows from (a).

(c) Define a second $Q(K H)$-action on $A$ by setting $a * \alpha=a g \alpha g^{-1}$ for all $a \in A$ and $\alpha \in Q(K H)$. By lemma 2(a), $(A, *)$ has the same submodules as $(A \cdot \cdot)$. Since $(A g, \cdot)$ is isomorphic to $(A, *)$ the result now follows.

The next lemma is just a variant of lemma 4 of Hartley and Richardson (to appear).

Lemma 10. Suppose $K G$ is semiprime and $H \leqslant G$ such that $[G: H]<\infty$. Then 


$$
\operatorname{soc} Q(K G)=\operatorname{soc} Q(K H) \cdot Q(K G)
$$

and

$$
\operatorname{soc} Q(K H)=Q(K H) \cap \operatorname{soc} Q(K G) .
$$

Proof. Suppose first that $\alpha \in \operatorname{soc} Q(K H)$ so that, by lemma 9(a) and 9(c), $\alpha Q(K G)$ is a finite direct sum of irreducible $Q(K H)$-modules. Hence $\alpha Q(K G)$ satisfies the minimum condition for $Q(K H)$-submodules, and so for $Q(K G)$-submodules. Since $Q(K G)$ is semiprime every minimal right ideal is a direct summand of $Q(K G)$. Hence $\alpha Q(K G)$ is a finite direct sum of irreducible $Q(K G)$-modules. Thus soc $Q(K H) \subseteq \operatorname{soc} Q(K G)$.

Now suppose that $\alpha=\alpha_{1} g_{1}+\cdots+\alpha_{n} g_{n} \in \operatorname{soc} Q(K G)$ where each $\alpha_{i} \in$ $Q(K H)$ and $g_{1}, \cdots, g_{n}$ is a transversal for $H$ in $G$. To show that each $\alpha_{i} \in \operatorname{soc} Q(K H)$ it is enough to suppose that $L$ is a large right ideal of $Q(K H)$ and show that each $\alpha_{i} \in L$. But then $L \cap K H$ is a large right ideal of $K H$ and so, by lemma 1(a), $(L \cap K H) \cdot K G$ is a large right ideal of $K G$. Hence ( $L \cap K H) Q(K G)$, and so $L \cdot Q(K G)$ too, is a large right ideal of $Q(K G)$. Hence $\alpha \in L \cdot Q(K G)=L g_{1}+\cdots+L g_{n}$ where this sum is direct. Equating the components of $\alpha$ in this direct sum with the components from the expression $\alpha_{1} g_{1}+\cdots+\alpha_{n} g_{n}$ then gives each $\alpha_{i} \in L$. Hence soc $Q(K G) \subseteq$ $\operatorname{soc} Q(K H) \cdot Q(K G)$.

Finally $Q(K H) \cap \operatorname{soc} Q(K G) \subseteq \operatorname{soc} Q(K H)$ follows from the previous paragraph with $g_{1}=1$ and $\alpha_{2}=\cdots=\alpha_{n}=0$. This completes the proof.

THEOREM 11. Suppose $H \leqslant G$ such that $G / H$ is residually finite. If $K G$ is nonsingular with uniform right ideals then

$$
K H \cap \operatorname{soc} Q(K G) \neq 0 .
$$

Proof. (Notice that we do not assume that $K G$ is prime.) By hypothesis soc $Q(K G) \neq 0$ so we choose $0 \neq \alpha \in K G \cap \operatorname{soc} Q(K G)$ and write $\alpha=$ $\alpha_{1} g_{1}+\cdots+\alpha_{n} g_{n}$ where each $0 \neq \alpha_{i} \in K H$ and $H g_{1}, \cdots, H g_{n}$ are distinct cosets of $H$. Since $G / H$ is residually finite there is a normal subgroup $N$ of $G$ of finite index in $G$ such that $N g_{1}, \cdots, N g_{n}$ are distinct and $N$ contains $H$. By lemma $10, \alpha \in \operatorname{soc} Q(K G)=(Q(K N) \cap \operatorname{soc} Q(K G)) \cdot Q(K G)$. If we equate the components for $\alpha$ given by this with the components of the expression $\alpha_{1} g_{1}+\cdots+\alpha_{n} g_{n}$ (possible because $N g_{1}, \cdots, N g_{n}$ are distinct) we find that each $\alpha_{i} \in \operatorname{soc} Q(K G)$. Hence, as each $\alpha_{i} \in K H$, we have $K H \cap$ $\operatorname{soc} Q(K G) \neq 0$.

COROLlary 12. If $K G$ is nonsingular with uniform right ideals and $H \leqslant G$ such that $G / H$ is residually finite and $Q(K H)$ is simple, then $Q(K G)$ is semisimple Artinian. 
Proof. By theorem 11, $Q(K H) \cap \operatorname{soc} Q(K G)$ is a nonzero ideal of $Q(K H)$ and so equals $Q(K H)$. Hence $1 \in \operatorname{soc} Q(K G)$ and the result follows.

If we look at locally finite factor groups instead of residually finite groups then lemma 10 does not seem to help us. Instead we use a dimension function on the lattice of finitely generated right ideals of $Q(K H) \cdot G$. This is a generalisation of the dimension function in Hannah and $O^{\prime}$ Meara (to appear), as may be seen by putting $H=1$ in what follows.

We suppose $H \leqslant G$ such that $G / H$ is locally finite and $Q(K H)$ is semisimple Artinian. Let $R=Q(K H)$ and $S=Q(K H) \cdot G$ and denote by $L(S)$ the lattice of finitely generated right ideals of $S$. If $X$ is an $R$-module let $\operatorname{dim} X$ be the composition length (= uniform dimension) of $X$. We define $d: L(S) \rightarrow[0,1]$ as follows: if $\alpha_{1}, \cdots, \alpha_{n} \in S$ choose a subgroup $N$ of $G$ such that $[N: H]<\infty$ and $\alpha_{1}, \cdots, \alpha_{n} \in R \cdot N=Q(K H) \cdot N$ and write

$$
d\left(\sum_{i=1}^{n} \alpha_{i} S\right)=\operatorname{dim}\left(\sum_{i=1}^{n} \alpha_{i} R \cdot N\right) / \operatorname{dim} R \cdot N .
$$

Proposition 13. If $H \leqslant G$ such that $G / H$ is locally finite and $Q(K H)$ is semisimple Artinian then $d: L(S) \rightarrow[0,1]$ is a well-defined function such that for any $A, B \in L(S)$

(i) $d(A)=0$ if and only if $A=0$,

(ii) $d(B)=1$ if and only if $B=S$,

(iii) if $A \cap B=0$ then $d(A+B)=d(A)+d(B)$,

(iv) if $A \leq B$ then $d(A) \leqslant d(B)$.

Proof. We note first that the expression for $d\left(\sum \alpha_{i} S\right)$ makes sense because $\operatorname{dim} R \cdot N$ is finite. That $d\left(\sum \alpha_{i} S\right)$ is independent of the choice of $N$ follows as in (2.1) of Hannah and O'Meara (to appear) by using lemma 2(b) and lemma 9(c). Independence of the choice of $\alpha_{1}, \cdots, \alpha_{n}$ then follows easily. Properties (i) to (iv) are consequences of the corresponding results for $\operatorname{dim} X$.

Lemma 14. Suppose $K G$ is nonsingular and $H \leqslant G$ such that $G / H$ is locally finite and $Q(K H)$ is Artinian. Then $Q(K H) \cdot G$ is semisimple and locally Artinian.

Proof. Obviously $Q(K H) \cdot G$ is locally Artinian. Since $K H$ is nonsingular, $Q(K H) \cdot N$ is right self-injective when $N$ is a subgroup of $G$ such that $[N: H]<x$ (by lemma $9(\mathrm{a})$ ). Hence the Jacobson radical and the (right) singular ideal of $Q(K H) \cdot N$ coincide. (See Faith (1967) page 47.) Thus the same is true for $Q(K H) \cdot G$ and so $Q(K H) \cdot G$ is semisimple.

THEOREM 15. Suppose $K G$ is prime nonsingular with uniform right ideals. 
If $H \leqslant G$ such that $G / H$ is locally finite and $Q(K H)$ is simple then $Q(K G)=$ $Q(K H) \cdot G$ and is simple Artinian.

Proof. It is enough to show that $S=Q(K H) \cdot G$ is simple Artinian. By lemma 14, and by lemma 2.1 of Fisher and Snider (1974), every nonzero right ideal of $S$ contains a nonzero idempotent. Since $S$ has uniform right ideals we deduce that $\operatorname{soc} S \neq 0$. Let $A \neq 0$ be a minimal right ideal of $S$. For any nonzero right ideal $B$ of $S$ we have $A \leqslant B$ since $S$ is prime. Hence $d(A) \leqq d(B)$ and so $d(A)$ is the minimum nonzero value attained by $d$. By proposition 13 it follows that a family of independent nonzero right ideals of $S$ cannot have more than $1 / d(A)$ members. Hence $S$ has finite uniform dimension. Being a prime Goldie ring with nonzero socle $S$ must be simple Artinian, as required.

Remark. The equality $Q(K G)=Q(K H) \cdot G$ attained in theorem 15 seems unusual. If we put $H=1$ we cannot have $Q(K G)=Q(K H) \cdot G$ as long as $K G$ is nonsingular and $G$ is infinite because then $K G$ is not self-injective. Yet, by theorem 15, if $K G$ is prime and has uniform right ideals then the equality does hold. An example of such a situation is as follows.

Let $A=\left\langle x, y: x^{-1} y^{2} x=y^{-2}, y^{-1} x^{2} y=x^{-2}\right\rangle$ and let $B$ be the subgroup of $A$ generated by $x^{2}, y^{2}$ and $(x y)^{2}$ (see Passman (1974), page 96). Let $G=\Pi_{l} A$ where $I$ is some infinite index set and let $H=\Pi_{I} B$. Since $A$ is torsionfree supersoluble $K G$ is an Ore domain and so certainly prime nonsingular with uniform right ideals. But $H$ is an abelian normal subgroup of $G$ with $G / H \cong \Pi_{1} Z_{2}$ an infinite locally finite group. By theorem 15, $Q(K G)=$ $Q(K H) \cdot G$ which is the ring of fractions of $K G$ with denominators the nonzero elements of the commutative domain $K H$.

\section{Applications}

Using the above results we shall now construct further classes of groups $G$ such that $Q(K G)$ is simple Artinian whenever $K G$ is prime nonsingular with uniform right ideals. Thus it is shown that $F C$-soluble groups and radical groups have this property (propositions 18 and 19) while, if we restrict our attention to the case when $K$ has zero characteristic, then linear groups also share this property (proposition 21).

We begin by looking at groups with a (seemingly) stronger property. Let $C$ be the class of all groups $X$ such that, whenever $K G$ is prime nonsingular with uniform right ideals and $H \triangleq G$ such that $Q(K H)$ is simple and $G / H \cong X$, then $Q(K G)$ is simple too.

In particular if $G \in C$ and $Q(K G)$ is a full-linear ring then $Q(K G)$ is 
simple Artinian. It follows from theorem 1.7 of Hannah and O'Meara (to appear) that if $G \in C$ has only countable conjugacy classes and $K G$ is prime nonsingular, then $Q(K G)$ is simple regardless of the presence or otherwise of uniform right ideals. By the results of the previous section $C$ contains all residually finite and all locally finite groups. More complicated groups can be constructed using the following result.

LEMMA 16. If $X$ is a group with an ascending series whose factors all lie in $C$ then $X \in C$ (that is. $C$ is $P$-closed).

Proof. By corollary 6 the proof (by transfinite induction on the length of the series for $X$ ) is trivial except possibly at limit ordinals. Suppose $K G$ is prime nonsingular with uniform right ideals and $H \unlhd G$ such that there is an ascending series $H=H_{0} \geqslant H_{1} \ni \cdots \geqslant H_{\lambda} \geqslant \cdots \geqslant H_{\mu}=G$ where $\mu$ is a limit ordinal and, for each $\lambda<\mu, Q\left(K H_{\lambda}\right)$ is simple Artinian. Since $K G=$ $\bigcup_{\lambda-\mu} K H_{\lambda}, \bigcup_{\lambda{ }_{\mu}} Q\left(K H_{\lambda}\right)$ is a quotient ring of $K G$ (by lemma 1) and, being a union of simple rings with the same identity, is simple. Hence $Q(K G)$ is simple too.

LFMma 17. Any torsion-free abelian group belongs to $C$.

PROOF. Let $K G$ be prime nonsingular with uniform right ideals and suppose $H \leqslant G$ such that $G / H$ is torsion-free abelian and $Q(K H)$ is simple. It is enough to show that $Q(K H) \cap \operatorname{soc} Q(K G) \neq 0$ and for this we may suppose that $G / H$ is finitely generated. But then $G / H$ is residually finite and theorem 11 completes the proof. (In fact it is not too hard to show that any uniform right ideal of $K H$ generates a uniform right ideal of $K G$ but we do not need that here).

Proposition 18. C contains all FC-soluble, all FC-hypercentral and all hyperabelian groups.

Proof. Since an FC-group has a locally finite normal subgroup whose factor group is torsion-free abelian. the result follows from lemma 17 and theorem 15 by using lemma 16.

PROPOSITION 19. C contains all radical groups.

ProOf. Suppose $K G$ is prime nonsingular with uniform right ideals and $H \leqslant G$ such that $G / H$ is radical and $Q(K H)$ is simple. By lemma 16 we may suppose $G / H$ is locally nilpotent. Choose $0 \neq \alpha \in K G \cap \operatorname{soc} Q(K G)$ and let $H_{1}=\langle H, \operatorname{supp} \alpha\rangle$. For any finite subset $X$ of $G, H_{1}$ is subnormal in $\left\langle H_{1}, X\right\rangle$ since $G / H$ is locally nilpotent. By lemma 1 and corollary $6 Q\left(K H_{1}\right) \subseteq$ $Q(K G)$ and is a full linear ring. But $H_{1} / H$ is nilpotent and $Q(K H)$ is simple. 
By proposition $18, Q\left(K H_{1}\right)$ is simple and since $Q\left(K H_{1}\right) \cap \operatorname{soc} Q(K G) \neq 0$ the result follows.

For our final result we need the following easy lemma.

LEMMA 20. If $G$ contains a noncyclic free subgroup then $Q(K G)$ cannot be semisimple Artinian.

Proof. If $H$ is such a subgroup then $K H$ is a non-Ore domain and so has an infinite family of nonzero independent right ideals. Hence so does $K G$ and so $Q(K G)$ is not Artinian.

Proposition 21. Suppose $K G$ is prime nonsingular with uniform right ideals and $H \leqslant G$ such that $G / H$ is linear and $Q(K H)$ is simple Artinian. If char $K=0$ then $Q(K G)$ is simple Artinian.

Proof. If $G / H$ is soluble-by-locally-finite then the result holds regardless of char $K$ (by proposition 18 and theorem 15). Otherwise $G / H$ must contain a noncyclic free subgroup by theorem 10.17 of Wehrfritz (1973). Hence $G$ contains a noncyclic free group. Say $x, y \in G$ such that $\langle x, y\rangle$ is free but not cyclic. Choose some nonzero $\alpha \in K G$ such that $\alpha K G$ is a uniform right ideal and let $N$ be the subgroup of $G$ generated by $H, x, y$ and $\operatorname{supp} \alpha$. Then $\alpha K N$ is a uniform right ideal of $K N$ and, because char $K=0, K N$ is nonsingular by theorem 4 of Snider (to appear). But $Q(K H)$ is simple Artinian and $N / H$, being a finitely generated linear group, is residually finite (by theorem 4.2 of Wehrfritz (1973)). By corollary 12, $Q(K N)$ is semisimple Artinian and, since $N$ contains a noncyclic free group, we have a contradiction. This completes the proof.

The above results show that for several large classes of groups the maximal right quotient ring of a group algebra cannot be an infinite dimensional full-linear ring. It is perhaps too soon to conjecture that this is true for all groups but $I$ have been unable to construct a counterexample. A related problem is that of determining when group algebras have nonzero socle: if a prime group algebra had nonzero socle its maximal right quotient ring would be an infinite dimensional full-linear ring. However Richardson (to appear) has conjectured that the group algebra of a non-locally-finite group has zero socle. In particular (by theorem 5) this would mean that prime group algebras always have zero socle.

Finally we summarise what little is known about the existence of uniform right ideals in group algebras. All previous work (that I know of) has concerned itself with determining whether a group algebra is an order in an Artinian ring. A necessary condition for this to happen is that the group contain no infinite locally finite subgroups (see Hughes (1973)). The weakest sufficient condition known seems to be the existence of an ascending series 
whose factors are all cyclic, and only finitely many of which are finite (see Hughes (1973) once more). But as the example at the end of section 2 shows, this restriction on the number of finite factors is still too strong. Also related to all this is the result of Lewin (1972) which states that if $G$ is soluble and $K G$ is a domain then $K G$ is an Ore domain (and so $K G$ is itself uniform).

Note: We have just learnt that $\mathrm{K}$. Brown has apparently also proved proposition 7. For more results on the equality $Q(K H) \cdot G=Q(K G)$ (see the remark after theorem 15) the reader should consult Lawrence and Louden (submitted), and the author's paper "Quotient rings of subgroup algebras" (submitted).

\section{Acknowledgement}

The author would like to thank his supervisor, Dr. K. C. O'Meara, for his help and encouragement.

\section{References}

W. D. Burgess (1969), 'Rings of quotients of group rings', Canada J. Math. 21, 865-875.

Carl Faith (1967), Lectures on Injective Modules and Quotient Rings (Lecture Notes in Mathematics, 49. Springer-Verlag, Berlin, Heidelberg, New York, 1967).

Joe W. Fisher and Robert L. Snider (1974), 'Prime von Neumann regular rings and primitive group algebras', Proc. Amer. Math. Soc. 44, 244-250.

Edward Formanek (1974). 'Maximal quotient rings of group rings', Pacific J. Math. 53, 109-116.

David Handelman and John Lawrence (1975), 'Strongly prime rings', Trans. Amer. Math. Soc. 211, 209-223.

John Hannah and K. C. O'Meara (to appear), 'Maximal quotient rings of prime group algebras', Proc. Amer. Math. Soc

B. Hartley and J. S. Richardson (submitted), 'The socle in group rings'.

Ian Hughes (1973), 'Artinian quotient rings of group rings', J. Austral. Math. Soc. 16, 379-384.

- R. E. Johnson (1961), 'Quotient rings of rings with zero singular ideal', Pacific J. Math. 11, $1385-1392$

John Lawrence and Kenneth Louden (submitted), 'Rationally complete group rings'.

Jacques Lewin (1972), 'A note on zero divisors in group-rings', Proc. Amer. Math. Soc. 31, 357-359.

D. S. Passman (1974), 'Advances in group rings', Israel J. Math. 19, 67-107.

J. S. Richardson (submitted), 'Group rings with nonzero socle'.

Derek J. S. Robinson (1972), Finiteness Conditions and Generalized Soluble Groups, Part I (Ergebnisse der Mathematik und ihrer Grenzgebiete, 62. Springer-Verlag, Berlin, Heidelberg, New York, 1972).

Robert L. Snider (to appear), 'On the singular ideal of a group algebra', Comm. Algebra.

B. A. F. Wehrfritz (1973), Infinite Linear Groups. An account of the group-theoretic properties of infinite groups of matrices (Ergebnisse der Mathematik und ihrer Grenzgebiete, 76. Springer-Verlag, Berlin, Heidelberg, New York, 1973).

A. E. Zalesskir (1973), 'On the semisimplicity of a modular group algebra of a solvable group', Soviet Math. Dokl. 14, 101-105.

Department of Mathematics,

University of Canterbury,

Christchurch,

New Zealand. 